\title{
Primary Discussion on the Ecological Garden Landscape Construction in the Cities
}

\author{
Xiao Qianwen \\ Hubei University of Technology \\ Hubei University of Technology, Hongshan District Wuhan, Hubei Province
}

\begin{abstract}
With the rapid development of the economy in China, the ecological problems in the cities in China are more and more severe. Due to the increase of globalization process, the urbanization process is also more and more rapid. All the countries all discuss and study the ecological environment, and China is included as well. How to improve our existing ecological environment and how to reach an excellent circulation have been an important topic that Chinese society faces now. Starting from the excellent circulation of the overall economy and make comprehensive study and discussion under the framework of garden landscape in cities, it can make our city ecological environment into a healthy track of initiative development, sustainable development and scientific development.
\end{abstract}

Key words-garden landscape in cities; recyclable

economy; ecological environment

\section{INTRODUCTION}

As is known to all, the urbanization speed in China is amazing. Cities are full of many upright buildings, broad roads and streets together with steels, cement, asphalt, dust, waste gases, and horns as well as various polluted gases and noise. People flock into cities and the population of per unit increase greatly and the rapid flow of urbanized population is increasingly fast. People gradually stay away from the nature environment which they should live with and squeeze into the limited room in cities. Every day, they are indulged in the dull building. In order to stay away from this unfavorable environment, the ecological landscape construction planning of the whole city should be taken as the key of the urban construction. However, people have to face the fact that the ecological landscape construction is far behind the construction of buildings. Besides, with the specific feature of cities, there are noises, traffic jams, shortage of houses etc. Facing with this situation, people have to attach the key importance on the construction of the urban ecological environment that are faced and touched by people so as to change the unfavorable environment that people live in with the scientific construction of garden landscape. Protecting ecological environment, developing recyclable economy and constructing ecological garden city have been the main problem that the society confronts with now. This paper discusses the several aspect of urban ecological garden construction so as to have a fully and in-depth understanding of it; in this way, it can promote us more actively construct the urban ecological garden landscape and make the urban ecological garden planning towards a healthy development.

\section{Several basic features of ecological garden city}

In the 1980s, the National Ministry of Construction proposes the concept of "Ecological Garden City". At first, as a creative activity, it aims at pointing towards a struggling direction for the urban construction. Ecological garden city requires the mutual sustainable development of economy and ecology whose construction is diverse and complex and various aspects should be taken into consideration, such as the geographic location, population, areas, advantages and disadvantages of the surrounding nature environment. Therefore, in a word, it can be understood as the ecological gardenization of natural landscape, such as mountains, rivers, lakes etc in the whole construction district of the city, including transport network, wetland, greenland, residences, business district, economic development zone, Industrial and mining zone, urban plaza, urban parks etc; all the above should be included into the overall planning for the natural ecological environment so as to realize the urban gardenization. Therefore, the urban gardenization refers to many disciplines and many art fields. Then its features should be interpreted as the following aspects: 


\section{Basic features of ecological garden city}

(1) Ecological garden city pays attention to the ecological position of the forest.

As the main body of ecology, forest is of great importance in the ecological system. The disappearance of forest means that people will have to face survival crisis and the human beings would extinct as well. Therefore, the ecological garden city paying attention to the ecological position of forest has been the only and necessary step. Some prophet once said that the last drop of water would be the tears of human beings; therefore, the ecological function of forest is quite important. Citizens cannot live without the planning and design of forest. The ecological development of city cannot do without the planning and design of forest. So forest can be said as the basic features of ecological garden city as well as important symbol of urban ecological garden.

(2) The ecological garden city attaches importance to the function and position of the overall ecology

The construction of urban ecological garden is not purely for the rest and leisure time of citizen. What is more important is that it can eliminate, digest and improve the series of environment pollution produced by urban social life. Only plants can do photosynthesis, release fresh oxygen and absorb carbon dioxide so as to purify the air pollution in cities. Therefore, the basic feature of ecological garden cities is not only to satisfy the aesthetic appreciation of citizen; what is more important is to build up the natural ecological environment which is beneficial to the living and social development of human beings, which is with picturesque scenery, fresh air as well as diverse green space for creatures in natural ecology. Therefore, paying attention to the function and position of overall ecology is the important feature of ecological garden city.

(3) Ecological garden city pays attention to the humanities status of ecology

The harmony of human and nature is another feature of ecological garden city. Respecting nature is respecting the development of human beings and following the rules of nature is loving nature. Every citizen is a member of nature ecology. Ecology should highlight the humanistic features of ecological garden, just as proposed in Shanghai Expo "Better City, Better Life", fully understand that "green hills and rivers is treasures left for the following generations".

2. Notes for construction of ecological garden city

(1) Less pollution. During the construction of ecological garden city, the first is to minimize the pollution. For example, the waste water is not drained directly but for recyclable use after better handling so as to reduce the pollution of waste water for cities in source. Another example is that human beings would produce many wastes and the typical is the waste battery, which is for recyclable use and its pollution for the whole ecology is minimized as more as possible.

(2) Rationality. This is represented in the construction of urban ecological garden should take all aspects into consideration so as to make a survey for the city and attention should be paid to every aspect in the city and build up a garden that suits the city so as to present its rationality. (3) Harmonization. The so-called harmonization obviously refers to the harmony between the city and the ecological environment of the whole city as well as the heaven and human so to reach a sustainable development.

\section{The principle of ecological garden city construction}

1. The economic construction-based principle

It is obvious that the development of a city cannot do without economic construction; it is the same that the urban ecological garden construction cannot do without economic construction; they are relative. Only on the basis of good economic construction, it has the capital to construct ecological garden city. The economic construction and ecological garden construction are complementary. Therefore, the success of ecological garden construction should be combined with economic construction and on the basis of economic construction-oriented so as to realize the planning of garden construction.

2. The principle of following nature development laws

Each thing has its essential features, which should not be violated by us. People must obey its natural development direction and combine with the nature features of things so as to achieve the success of urban garden construction. Natural development law is conferred by nature; if people 
violate it, then the urban garden construction must be a failure and the final result would not be satisfactory.

3. The principle of emphasis on actual effect

The reconstruction of the cities people live can make it towards the direction of ecological urban garden, which is not for the so-called beauty or the urban images. Finally, the ultimate purpose is for the comfort in the cities and improvement of our living quality. For this purpose, people should pay attention to the final effect of ecological garden for the real benefits of human beings.

\section{How to build up the urban ecological garden landscape}

1. The construction of nature corridors

Natural corridor can be divided into three aspects, namely the river corridor, mountain corridor and artificial corridor, which makes up the keys of ecological urban construction. The whole development of the city develops from inside to outside, which violates the development of whole nature. Therefore, river corridors are necessary for the dredging of cities and release the relevant pollution, traffic and populations of the city. The construction of river corridors cannot only reduce river pollution, connect city and nature; it can also keep the quality of river so as to form an effective and better ecology. Therefore, the construction of river corridor is indispensable. The second is the construction of mountain corridor. It is not hard to imagine that river corridor has been one part and the construction of mountain is also necessary. Mountain has a great advantage of high green plants coverage, which is the source of city oxygen. Therefore, in order to better transmit these extra carbon dioxide, mountain corridors are necessary. Then the last is the key of ecological garden construction, namely artificial corridor. The reasonable traffic hubs can make the city unimpeded and convenient for the emission of city pollutants, such as noises, waste gases and wastes. At the same time of construction for artificial corridor, it should pay attention to the artificial construction of plants. For example, it should select some anti-pollution plants or plants with strong survival ability. The second is the well-ranged of these plants so as to reach the beautiful effects of the city, which is ordinary in our daily life. These ecological garden constructions are more and more, which makes us happy.

2. The construction of plaque

The construction of plaques consists of several aspects, such as urban plaques, suburb plaques and wetland plaques. Firstly, the urban plaques mainly consist of buildings, parks and streets. In order to achieve a better ecological effect, people should pay attention to the construction of parks and leisure nature spaces. Intervene our living spaces with more nature landscape and change the urban building. Secondly, the suburb plaque mainly consists of villages, lands, mountains and rivers. Some suburb tourism sceneries should be developed as much as possible. Combined with natural conditions, plan artificially the suburb construction. The last is the construction of wetland plaques. Therefore, the combination of human and things, people should pay attention to the development of wetland, such as some boards of wetland parts so as to utilize the wetland into the urban construction.

\section{$\mathrm{V}$ Improving the effect of ecological garden city and} increasing the aesthetic value of landscape

The construction of ecological garden city lies in the construction of ecological garden. The orientation of ecological garden construction should increase the greening space of city and increase the green space of city. Just as is known to us, green plants can absorb the waste gas in the city, such as carbon dioxide and produce oxygen. This fresh air should attribute to green plants. After the fresh air, the living environment is better. What are more important, green plants can stimulate our retina and protect our eyesight. Apart from work, green plants can release our tiredness. Therefore, in order to improve the greening rate of city and build the ecological garden, it is helpful for our study and work.

\section{Modes for the ecological design of urban garden landscape}

\section{Tourism modes}

With the rapid development of economy in China, people not only pay attention to food and clothing but more attention on spiritual needs. Therefore, in order to meet this demand, it can make some contribution on the garden 
landscape construction. In order words, the urban ecological planning design should take the tourism development into consideration and involve all the aesthetic process and urban ecological garden landscape into one.

\section{Cultural patterns}

During the construction of ecological garden city, apart from the aesthetic aspect, it should involve with the cultural features of the city. And it should retain the cultural features of the city. In this way, the ecological garden construction has its own features and makes each city unique.

\section{Great importance for urban ecological garden construction}

1. The emergency for ecological garden city construction

As is known to all of us, the living environment is more and more severe with much waste gas, waste water and wastes. Living in such environment, people should save it. After long-time trying and exploration, human beings try a lot, even live in outer space. However, in actual situation, the most realizable is to construct the current survival environment, or the construction of ecological garden city. With the rapid development of economy, construction of ecological garden city has been an emergency. So people should take action and make this into the construction of each city. The stupid method for pollution first and then control should be avoided. People should have their attitudes and envisage the current problem so as to strive for the actual sustainable development.

2. The construction of ecological garden city is helpful for the sustainable development of harmony society

The main aim for construction of ecological environment is to improve our living urban environment and improve people's life quality as well as improve the pollution, noises and traffic problems so as to provide a more comfortable living environment. People advocate the harmony of society, which refers to harmony among human beings, harmony between human and nature. Construction of ecological garden is to make the city soft and full of energy and return the intimacy. Therefore, combined with the specific features of city, the constructed ecological garden can combine it together and make nature in human's heart, which can undoubtedly realize the sustainable development. Then the sustainable development concept can effectively presented in the construction of ecological garden city.

\section{Conclusion}

In a word, the construction of ecological garden city is not and coincides and it has the necessity. It appears with the development of human society and the development of historical process. In the long river of history, the city experiences much recreation which all follows the development of society. With the increase of people's living standard and concepts, people also have some new opinion and viewpoints to the urban construction. In order to solve this realistic problem, people put forward the sustainable development concept, which is of great importance to the ecological garden construction. In the construction process, people should fully consider the features of cities and recreate with its features, which can realize the comfort and harmony of nature environment and provide stimulus for the social and economic development.

\section{Acknowledgement}

Project: Human Studies and Social Science Research Plan of Education Bureau

Project NO.:15YJA760035

\section{References:}

[1] Yi Fengquan, Zhang Yaosheng \& Zhang Duanlan. Discussion on the greening construction by the garden plants resources $[\mathrm{J}]$. Journal of Anhui Construction Industry Institute (Natural Science Edition) .2005, 03.

[2] Zhu Youjie. Thoughts on the forming, development, quality and specific functions of aesthetics [A]. Essay collections for the history and development of landscape garden discipline [C]; 2006.

[3] Gao Yaguang, Wang Sa, Wang Hongkui \& Wang Qi. Study on the application of city vertical planting in the garden application [A]. Agroforestry production technology symposium on innocuity in Liaoning Province[C], 2011. 